\title{
Ten arguments for a societal perspective in the economic evaluation of medical innovations
}

\author{
Bengt Jönsson
}

Published online: 19 July 2009

(C) Springer-Verlag 2009

Health technology assessment (HTA) is increasingly used to assist decisions about reimbursement and funding of new medical technologies, particularly drugs. This means that the economic evaluation that is part of an HTA will form the core element of an assessment used for guiding decisions on resource allocation. While HTA in general has a societal policy perspective, many HTA and reimbursement agencies advising payers take a narrow budget perspective on the impact on resource use when performing economic evaluations. Examples of such agencies are the National Institute for Health and Clinical Excellence (NICE) in England and Wales, the Canadian Agency for Drugs and Technologies in Health (CADTH) in Canada, the Pharmaceutical Management Agency (Pharmac) in New Zealand, and the reimbursement agency NIHISB in Belgium. A number of initiatives for introducing or promoting the use of use HTA for health care policy making (IQWIG, EUnetHTA) seem uncertain about where to stand on the perspective of economic evaluation. There is also an important discussion about the consequences for pharmaceutical innovation of using cost-effectiveness studies to determine which technologies should qualify for reimbursement [1].

Ignoring important costs and benefits in an economic evaluation will lead to an inefficient allocation of resources, in the short term as well as from a long-term perspective. Cost-effective drugs will not be reimbursed and incentives for innovation will be adversely affected. The

B. Jönsson $(\square)$

Department of Economics,

Stockholm School of Economics,

Box 6501, 11383 Stockholm, Sweden

e-mail: Bengt.Jonsson@hhs.se role of HTA in establishing a transparent and efficient global market for medical innovations may therefore be questioned.

This paper provide ten arguments for taking a broad societal perspective on value, specifically to include all relevant costs, in HTA studies aimed at informing decisions about resource allocation. The purpose is to advocate that a broad perspective on value is necessary in order for the study to provide the correct incentives for decision makers to take into account, for both static and dynamic efficiency, when making decisions about allocation of resources for improvement of health. For a more in depth discussion, see [2].

1. A societal perspective is necessary for making optimal societal decisions.

Cost-benefit analysis - the theoretical model for costeffectiveness analysis-was developed to assess the desirability of projects from a societal perspective.

Cost-benefit analysis is a widely used technique of applied welfare economics, which is used to throw light on the societal desirability of undertaking an economic project. A project can be defined as an act of investment, introduction of a new commodity or a change in policy.

The new Palgrave Dictionary of Economics, Macmillan, 1987, p. 687.

Regulatory decisions about market authorisation of new medical technologies are based on an assessment of the benefits and risks from a societal perspective. HTA studies, in bringing in a wider perspective on the balance between potential costs and benefits, should take the same societal perspective since the objectives are the same, i.e. to promote the introduction and access to technologies with a potential high net benefit, and restrict others.

2. A societal perspective for economic evaluation is the classical approach to assessing the profitability of societal 
investments, also used for assessing health benefits from investments in other sectors of the economy.

This is for example the standard approach in the assessment of different environmental, and transport safety programmes affecting health. There is no reason why economic evaluation of programmes affecting health in the health care sector should deviate from this standard. Adopting a payer instead of a societal perspective will create a bias against investments in improved health through health care spending.

3. If health gains are valued from a societal perspective, so should costs. It has been widely accepted that economic evaluations should include all potential health effects, positive as well as negative (side effects).

It is illogical to take a societal perspective on health benefits but not on costs. Cost-effectiveness analysis can be performed within a specific budget perspective if outcome is services, not health (productivity analysis). Why should health effects be included in quality adjusted life years (QALYs), for example from a new effective treatment for dementia, but not the related reduction in costs for informal care used to compensate for lack of an efficient treatment? Both are equally important parts of the value of a new treatment.

4. A restricted payer perspective will lead to suboptimal decisions for allocation of resources, affecting both static and dynamic efficiency.

Economic evaluations based on a fixed budget may lead to suboptimal decisions. Switching costs to other parties may make an investment attractive, for example moving prevention from within towards outside the health care sector. What is within and outside the budget is a policy decision, making it an arbitrary decision what is included or not. Costs outside the budget period are not counted, making investments with a high fixed cost less attractive than those where costs are spread over a longer time. It is often difficult to verify to what extent a particular consequence impacts on the budget, if at all? For example, illness among health care workers may increase budget costs if temporary staff have to be hired at a higher salary. Costs for health care personnel are included in direct costs because there is an opportunity cost. When disease affects other workers, there is also an opportunity cost, and thus this should be included (indirect costs).

5. Empirical studies support the risk of suboptimal decisions based on restricted view of benefits.

Alzheimer's disease, multiple sclerosis and rheumatoid arthritis are examples of disease areas where major benefits from new innovations will come in terms of reductions in productivity gains and reductions in costs for community and informal care. Not counting these values of innovation will provide suboptimal use of treatments and incentives for innovation.
6. Payer perspectives cannot be defined in a consistent way without a societal perspective, and thus QALYs will not have a consistent definition either.

All health care costs, both now and in the future, including health care costs in added years of life, should be included. Most countries have several health care budgets, making it difficult to define a consolidated budget, particularly in regionalised health care systems. Which costs are included in which budget varies between countries and changes over time. Thus the principle must be to include all relevant costs, regardless of who pays for them. This was also the guiding principle when cost-effectiveness and cost-utility analysis was developed.

All changes in real resources should be measured, and can be classified as changes in service production, changes in resources used by patients and their helpers, and changes in the gross domestic product (Alan Williams [3], page 272).

7. It is problematic to measure and interpret QALYs if they include external costs.

It has been argued that including, for example, indirect costs involves double counting, since those effects are taken into account in the QALY measure. But this is unlikely since changes in incomes are, at least to some extent, compensated through societal insurance systems. Empirical evidence also supports the view that measures of QALYs do not include these effects [4]. For QALYs to be interpreted as a pure measure of health benefit, it is also important that productivity effects are explicitly excluded when the value of a health state is assessed.

8. A restricted budget perspective is inconsistent with decisions based on willingness to pay for QALYs.

The willingness to pay (WTP) for a QALY may vary over time and between diseases, groups of patients and the technology used. These different valuations have, and should have, an impact on budgets. Research on the "value of a QALY" is meaningless unless the cost per QALY ratio is clearly and properly defined, which means that if the cost definition changes over time and between decision makers, it is not possible to interpret the cost per QALY ratio.

9. Specific payer perspectives should be assessed within the societal perspective.

Within a societal perspective, it is possible to differentiate the perspective to address specific policy issues. It is difficult to know in advance which perspective is most relevant. A limited payer perspective is more interesting when related to the societal perspective, since incentives are determined by the distribution of costs and benefits. Investigating the distribution of costs and benefits over different stakeholders gives information for the design of policies for optimal decision making. 
10. A societal perspective supports an informed public discussion and democratic decisions, and facilitates international collaboration.

The HTA is not the decision-it is a tool designed to help make better decisions. In all countries, it is the population at large who both pays for and receives the benefits of new technologies. A broad societal perspective on value, i.e. costs and benefits, facilitates informed discussion and decisions about access and use of new medical technologies. Since medical innovation is a global public good, a societal perspective facilitates international collaboration in assessing new medical innovations, and helps coordinate national decisions about funding and patient access.

\section{References}

1. Jena, A.B., Philipson, T.J.: Innovation and technology adoption in health care markets. AEI, Washington, DC (2008)

2. Johannesson, M., Jönsson, B., Jönsson, L., Kobelt, G., Zethreaus, N.: Why should economic evaluations of medical technologies have a societal perspective? OHE Briefing Paper, London (2009)

3. Williams, A.: Welfare economics and health status measurement. In: van der Gaag, J., Perlman, M. (eds.) Health, economics and health economics, pp. 159-170. North Holland, Amsterdam (1981)

4. Davidsson, T., Levin, L.-Å.: Do individuals consider expected income when valuing health states? Int $\mathrm{J}$ Technol Assess Health Care 24(4), 488-494 (2008) 